\title{
Several Key Points in Early Human Innovative Evolution
}

\author{
Xu Guirong \\ Department of Paleontology, China University of Geosciences, Wuhan, China

\section{Email address:} \\ wwjjxu@163.com

\section{To cite this article:} \\ Xu Guirong. Several Key Points in Early Human Innovative Evolution. American Journal of Applied Scientific Research. \\ Vol. 4, No. 2, 2018, pp. 5-11. doi: 10.11648/j.ajasr.20180402.11
}

Received: July 22, 2018; Accepted: August 8, 2018; Published: September 5, 2018

\begin{abstract}
This article discusses the innovative evolution of biology. In order to supplement the evolutionary theory that emphasizes "adapting to the environment", we discuss the innovative evolution of "natural law". The first event in the universe to be observed was "innovation", and it was discovered that there were many innovations in human evolution. It introduces what is innovative evolution and what rules to follow. It focuses on the analysis of the performance of innovative evolution in human evolution. The innovative evolution of early human beings are mainly reflected in the liberation of hands in upright walking; the use of fire in artificial fire is the beginning of the active acquisition of energy and the active use of energy; the cooked food is fully obtaining nutrition that made the function of the brain perfect; the development of abstract thinking is the deducing interpreting substances and reforming these substances. This is the fundamental difference between human being and other animals. it is not possible for any animal to take the initiative to obtain energy and to produce food actively. So it is impossible for other primates to evolve into humans.
\end{abstract}

Keywords: Innovative Evolution, Early Humans, Artificial Fire, Cooked Food, Abstract Thinking

\section{Overview}

Darwin [1-2] published the Origin of species (1859), and in 1871 published the book the Origin of Man and the Choice of Sex, which broke through the heavy resistance and put forward the idea of "changing from ape to man". Now science has fully proved Darwin's view of evolution to be correct.

Two facts support Darwin's view of evolution: (1) fossil data; (2) molecular biology, namely DNA analysis.

\subsection{Fossil Data}

Since Linnaeus 1758 was identified Homo Linnaeus, a large number of fossils of the genus Homo Linnaeus have been discovered. [3-7]

Known to belong to the genus including Homo ergaster Grovers and Mazak, 1975, Homo erectus [Duboisi 1892], capable people (H. habilis L. S. B. Leakey et al. 1964), Homo heidelbergensis Schoetensackin, 1908, Homo neanderthalensis Kings, 1864, and the Lodofis (H. rudolfensis Alexeev, 1986) and Homo sapiens (H. sapiens Linnaeusus 1758). The Sinanthropus pekinensis, discovered in Zhoukoudian, Beijing in 1929, should also be classified as the genus of man [8].
Hominidae are divided into two subfamilies: Hominines includes the genus Homo [9-11], and the subfamily Australopithecines, includes four genera.

The fossil data clearly show the evolution of Homo sapiens [12-13].

\subsection{Biomolecular}

The anatomical characteristics of humans and primates are essentially the same, and genes and proteins are largely the same. Humans and chimpanzees share at least $98.4 \%$ of their DNA [14-15]. According to media reports, chimpanzees share 98.7 percent of the human DNA sequence.

The difference between humans and chimpanzees from body shape to habits, especially intelligence, is so great that the genetic difference is only 1.3, and many scholars are puzzled. Some scientists point out that the study underestimated the difference between human and chimpanzee DNA sequences, arguing that there is at least 5\% difference in DNA sequence, and the difference is not only in sequence, but mainly in function. 


\section{Evolution of Biological Innovation Is a Natural Law}

\subsection{The Meaning of the Term "Innovation"}

What is "innovation"? The word "innovation" or "creation" often causes misunderstanding in biology, is misunderstood as a reprint of "creation", or is idealistic. But this word is not the patent of Genesis after all, because there is "innovation" everywhere in the material world, as well as "innovation" in the biological world. Here is a metaphor to illustrate the meaning of "innovation". According to astronomical knowledge, there were only hydrogen atoms in the early days of the universe. Under certain conditions, hydrogen atoms were synthesized into helium, as is now happening in the sun. The production of helium is the innovation of nature. And a variety of other elements and molecules, including organic molecules. The first appearance of molecules, nucleotides, RNA or DNA, for example, is innovative, and all organic macromolecules, whether from the universe or from the earth, are clearly innovative in nature. The term " innovation " is used in this sense. The term " innovation " seems difficult to be attributed to " natural selection " or " adaptive environment ". Some scholars say that nature is chosen to be stable, so they can exist, but the original stable hydrogen then becomes stable helium, which is an innovation process. It is not a selective process $[16,17]$.

\subsection{The Rules of Biological Innovative Evolution}

Biological innovative evolution are conditional, and this condition is the law of nature. Innovation in biological evolution is carried out under the stimulation of natural matter and the induction of natural laws. Various physical properties and chemical substances in nature, such as different gravity, various elements and molecules, and various physical properties, including light, electricity, sound and radiation, are stimulating living things all the time. Under the constant stimulation of natural matter, organisms take the step of "innovation", and the induction of natural laws is the key to innovation. For example, the formation of chlorophyll molecules must follow the characteristics of light waves and the rules of elemental composition molecules under the stimulation of light. This can be called "The principle of stimulation and induction", each "co-innovative evolution" have its own specific stimulus and induction content.

For example, families often use the method of "stimulation and induction" to educate their children, and the method of "forcing with a big stick" is not good enough to guide their children correctly. To learn well, a child must be told repeatedly that the importance of learning is to constantly "stimulate" his brain; then he needs to follow the lead. If the child already understands, you still blindly "stimulate" without the "inductive" method, may be counterproductive, make the child resentful. At this time, the most important is to guide children to set up the correct learning methods and make concrete help, so that children can make great progress in learning.
The principle of "stimulation and induction" requires conditions for action. Organisms themselves must have a certain material basis to respond to external stimuli, such as the existence of organic molecules capable of absorbing light waves, in order to develop to chlorophyll molecules. At the same time, there is a good external environment, such as not too low or too high temperature except light, which is the necessary external condition for the formation of chlorophyll molecules. This is the law of "internal and external combination" followed by collaborative innovation. The structure and mechanism of biology and the laws of natural physical chemistry control the innovation of biology [18-20].

\section{The Main Key Points of Human Innovative Evolution}

\subsection{Walking Upright}

The vertical walking leaves a trace on the bones. It can be seen from the fossils of Homo erectus that the vertebrae is upright or nearly erect, the pelvis is directly on the leg bone, the tailbone shrinks or disappears, and the skull and the neck bone are nearly straight line.

Some of these characteristics are inherited from apes and are mostly the result of the development of upright walking [21-23].

Walking upright can support large-capacity skull and provide support for the development of brain. Walking upright freed the forelimbs and laid the foundation for further evolution of the hand.

Walking upright can be explained as adaptation to environmental changes, but then co-evolution with the hand, in order to work with the hand, the lower limbs and feet changed dramatically.

\subsection{The Creative Labor of the Hand}

The forepaws of forelimbs have five fingers separating, they has the grip ability is the hand basic characteristic. Apes in the climbing and picking activities such as grip strength greatly increased. In Homo erectus, the span of tiger's mouth between thumb and index finger increased, and the dexterity of five fingers increased with the increase of brain power.

Stone tools made by man are innovative labor and cannot be interpreted as adapting to the environment. Man according needs made all kinds of tools with wisdom, including stone tools, wood, pottery, bronze and iron. Every new invention is an innovation. The hand becomes the tool of creative labor [24-26].

The hand improves the hand itself, and improving the brain, the hind limbs, and the whole body.

Chimpanzees can also use and make tools, and U.S. and German scientists have reportedly excavated chimpanzee sites in the Coate d'Ivoire rainforest of West Africa, and found that chimpanzee stone tools are similar to those used by human ancestors. They dug up about 40 kilograms of nut shells and 479 stone debris. Chimpanzees use stones as hammers, tree 
roots as chopping boards, and to tunk nuts.

In the 1960 s, scientists reportedly observed chimpanzees tearing off their branches and making them into ant nests, to hook ants and appease hunger.

These chimpanzee activities are also innovative, but their activities are everywhere, no longer moving forward. Because their mental retardation is content to adapt to the existing life, no another innovation, so it is impossible to move forward.

\subsection{The Stimulation and Induction of Cooked Food Make the Human Brain Evolve Rapidly}

The forest fire gave Homo erectus cooked food. With the accumulation of experience, the temptation and stimulation of cooked food, it is an important event in life to expect to obtain the cooked food that is burned naturally.

\subsubsection{Cooked Food Promotes the Improvement of Nervous System}

The first direct exercise in cooked food is taste and smell. The conditioned reflex of barbecue aromas arouses a desire to eat; the experience of cooked food develops their sense of smell, allowing them to distinguish between different types of food. In this regard, Penny [27] asked whether comparing chimpanzees to humans' sensitivity to olfactory stimuli has improved the ability to accept smells as a result of an increase in the proportion of cooked foods in the diet.

Delicious taste deepened the early human cravings for cooked food, making them the first step to preserve the fire. At the same time, the vision and hearing are improved, the high contrast between the flame and the scorched food in search, the intense stimulation of the vision, the enhancement of the vision and the ability to distinguish the color and luster; the vocal connection in the collective search, the joy of finding the cooked food, exercise hearing and sound tape. The evolution of the five-facial organs promotes the rapid development of the brain.

\subsubsection{Cooked Food Promotes the Evolution of Human Brain [28]}

Under the combined conditions of labor, use of tools, social activities and language, the role of cooked food stimulation and induction in the evolution of the human brain can be summarized as follows:

a. Cooked food, easy to chew, canine teeth become smaller because they don't have to bite hard. Chewed food and saliva mix, with relish easy to swallow, oral volume also become smaller. Space is provided for the development of brain capacity. The brain volume is gradually increasing.

b. Chewed cooked food is easier to digest and absorb. After eating, the blood entering the stomach and intestines decreases relatively, and more abundant blood flows to the brain. Adequate nutrition of brain cells at any time creates conditions for the improvement of the function of brain nerve cells. Because the human brain is a very energy-consuming organ, modern brains account for only about 2 percent of the body weight, but consume away 20 percent of the energy. Therefore, a rich supply of nutrients is necessary for brain development.
The improvement of metabolism promotes to acquire rich nutrition [29].

c. Improvements in food hygiene extend life expectancy, allow enough time to accumulate experience, and be passed on to future generations. The resulting brain habits and improvements in brain function are inherited and enhanced for generations.

d. Pursue cooked food to improve taste, smell and vision overall. Promote the creation of tools using the tool labor, through creative labor to accelerate the evolution of the human brain.

\subsection{Artificial Fire, the Leap of Innovative Labor}

Natural fires often occur, initially after forest fires can be obtained cooked food, cooked food attraction, ape-man fire pursuit of cooked food. From picking natural barbecue food to making cooked food is an innovative mental development activity.

This is the key to entering Homo sapiens and marks a leap in the human brain. About 500,000 years ago finally learned to make a fire. In the Tapang Cave $24 \mathrm{~km}$ south of Haifa, Israel, Israeli scientists have discovered that flint deposited 350, 000 years ago shows obvious signs of burning [30]. this is evidence of human fire. Israeli scientists have recently reported that humans were able to use and control fire 790 , 000 years ago.

Richard Wrangham of Harvard University thinks our ancestors held fire earlier. He suggested that the ancients had begun cooking food about 2 million years ago, when they had evolved smaller teeth and the corresponding digestive system. Wrangham believed that the use of fire supported the evolution of many human characteristics, including the human huge brain. [31]

It takes about millions of years from finding natural cooked food to preserving fire to using tools to make a fire. The accumulation of long-term experience is the main way to enhance memory; the successful use of tools and artificial fire enables them to learn to think with their brains.

The fire is preserved for cooked food, but the stored fire is often extinguished and cannot stably obtain cooked food, and the natural source of fire is not always available everywhere. But apes are already familiar with experience, from the trees to get fire, from the impact of stone tools to see the flying flare. Drilling wood may be an early way to make fire. The legend of "Suiren's drilling for fire" is widely spread in China. Later, the firing of stone, especially the use of flint with nitrite, and even the use of sunlight to make fire. The temptation of cooked food led them to try every possible way to build a fire, using the method of rubbing flint or drilling wood, after a long period of effort, to create the method of artificial fire.

To learn to make a fire is the beginning of man's overcoming the complete bondage of nature. The teaching of fire building method, domestication of domestic animals and cultivation of crops, accumulation of knowledge in all aspects, brain as the main means of survival, has played a role in promoting the development and evolution of the brain.

Artificial fire is a decisive step in human innovative 
evolution, completely different from other primates. Fire can be used to process food, improve physique, and reduce disease. Fire can be used to keep warm, especially in the ice age, in order to resist the cold, heating with fire becomes inevitable. Fire can banish the invasion of the beast and protect itself. Humans who learn to catch fire can move from the tropics and the temperate to the cold, and their footprints begin to reach previously difficult places. Heating by fire also gradually degenerated body hair into naked apes, becoming true people.

\subsection{The Brain Volume Increases and the Brain Function Gradually Improving}

The constant use of the brain increases the brain volume. The increase in the amount of brain volume in the upright position is no longer a heavy burden. In the evolution of ape-man, the brain capacity changed from small to large. Compared with apes, such as the Alpha Australopithecus (a skeleton and skull containing 40 percent found in the Afa region of Ethiopia in 1974), is about 3.5 million years old and has a brain capacity of about $400 \mathrm{~cm}^{3}$ [32]; The brain volumes of the modern gorilla, chimpanzees and gibbons are also about the size of $400 \mathrm{~cm}^{3}$. Fossils of the Neanderthals were found in the Neanderthal Valley of Germany in 1857. As early Homo sapiens, their brain capacity was $1450 . \mathrm{Cm}^{3}$ is larger than the average brain size of modern humans (about $1400 \mathrm{~cm}^{3}$ ), and the Cro-Magnons with a brain volume of $1700 \mathrm{~cm}^{3}$ in the same period found near the village of Les Eyzies, France..

In fact, the evolution of the brain is not only in the brain capacity, but the increase in brain capacity is the initial manifestation of the evolution of the brain. Human brain evolution is mainly manifested in the number and function of the brain cells. The number of human brain neurons is close to the number of Milky Way stars, more than 15 billion. The access, transfer and processing of information by the human brain's cerebrum cells degree is an incomparable [33]. to any other animal.

Why did the Neanderthals and the Cro-Magnons with large brains die out, speculating that they failed to develop cooked food, that large brains could not be enriched, and that they were later wiped out by Homo sapiens.

Engels [34] (1876) once said: From eating plants to eating meat at the same time, and this is an important step in the transition to people. Meat food is almost ready to contain the most important material necessary for the body's metabolism; it shortens the digestion process. Thus, more time, more material and more energy to live a real animal's life. but the most important thing is the effect of meat on the brain marrow, which has been given much more of the material necessary for its nutrition and development than in the past, so that it can develop faster and more fully in generations.

But eating raw meat and drinking blood can not promote the rapid development of the brain. Many of the predators at the top of the food chain are primarily carnivorous, and their brains are still low. After eating their prey, the tiger sleeps or slackens, and is lazy to catch even when it is near. Because bloody raw meat takes time to digest. After a full meal, they can not eat for a long time. Tigers and lions have large heads because of their need to hunt and bite raw meat, their mouths are large, their blood basins of macro-mouth occupy large spaces, and their brains are relatively small.

If Engels changed what Engels called "meat food" to "cooked meat," it would be easy to understand the rapid evolution of the brain. Some scientists have pointed out that an increase in the proportion of cooked meat in early human diets is important for increased brain volumes.

\subsection{Development of Abstract Thinking}

The fundamental difference between man and other animals is the abstract thinking of the human brain. The abstract thinking of the human brain stems from man's imagination after another death. In forest fires, in the burning of objects, smoke twines and rises slowly. Thousands of experiences of this vision have left a profound influence in the minds of ancient humans. This memory is repeated hundreds of times with associations of death, especially among relatives burned to death in forest fires. The sight of smoke on the burned remains of the same kind reminds them of their ascent after death. The Neanderthals and the Cro-Magnons have funeral customs that suggest that thinking about people after death was the earliest abstract thought.

With the extensive use of labor tools, the skillful use of tools, the thinking of the results after the use of tools, the longing for the results of labor after animal husbandry and cultivation, and the extensive communication of language, abstract thinking is gradually expanded. With the increase of abstract thinking, the function of brain cells becomes more and more perfect. With the development of auditory, visual, taste, olfactory and tactile nerves, the ability of brain thinking is enhanced.

\subsubsection{The Characteristics of Human Brain Innovative Evolution Can Be Summarized as Follows}

a. In the process of making tools, using tools and engaging in labor production, the subjective activity of labor makes the senses receive comprehensive stimulation, which is the main reason to promote the development of the brain. The stimulation of vision, taste, smell, hearing and touch in labor will inevitably increase and differentiate the brain cells and gradually improve the function of brain cells.

b. Increased sensory stimulation with the subjective initiative of fire, adequate nutrition for cooked food and communication of language, which contribute to the sound development of the brain nerve and the entire system, including sensing speed, under the overall stimulus received by the senses, Information processing, information feedback and response functions are gradually improved.

c. It is natural matter that induces the development of brain cells and nervous systems in the direction of logical thinking. The induction of the properties of materials such as light waves, sound waves, radio waves, shock waves and other radiation waves. These waves of matter leave a variety of marks on brain cells and nerve cells according to which information is transmitted and interpreted in the human brain.

$\mathrm{d}$. The mind of any human being is inextricably linked to 
physical existence, logical thinking, fantasy, invention, planning and prediction of the future. Human thinking and scientific creation can only operate within the limits of the laws of nature. All illusions, religions, and theology are created by man's long-lived experience. Scientific thinking is to make logical reasoning according to the laws of nature, and the thinking without matter does not exist.

\subsubsection{The Characteristics and Leaps of Human Brain Thinking}

Human sensory information is transmitted from the sensory nerve to the central nervous system, which enters the cerebral cortex and functions with the endogenous hormone system to form a memory and become conscious. The incoming consciousness includes visual information, auditory information, olfactory information, tactile, painful, warm and kinesthetic information. Hearing and vision quickly process incoming acoustic and optical information, which is carried out in an orderly manner, making it a temporal and spatial perception.

There are two causes for the formation of consciousness:

a. Intuition, sensory information from the nervous system, such as vision, hearing, smell, touch and so on, such consciousness is very clear, is a direct consciousness.

b. Abstract thinking, which accumulates and deduces the experience of direct consciousness, forms various concepts and logical reasoning, and is abstract consciousness. Abstract consciousness creates a series of associations through the communication of language and writing in social relations and becomes a systematic consciousness.

Thinking becomes subjective when it is far from the basis of sensory information. There is only one mode of thinking in the human brain, which is to deduce and deduce objective perception according to human accumulated experience. Dream is the product of abstract thinking, only human beings have it. Animals may also sleep, leaving traces of fruit picking and eating in their brain cells. These dreams are not abstract deductions, but merely remnants of memory. Human dream is not only simple memory, but also thinking deduction, which is the form of brain cell movement. Throughout history, many scientists and artists have answered certain questions in their sleep. Research proof The mental deduction of the mind in the sleep can break out the spark of thought, and urge the mind to reconstruct [35].

\subsection{Domestication and Cultivation}

Early humans hunted for a living and invented tools such as stick spears, bows and arrows, which played an important role in fending off beasts of prey. Hunting is often intertwined with repellent beasts, which, by virtue of their fast running and claw teeth, often outperform early humans. But the man who conquers the beast from the innovation thought, on the one hand is invents the powerful weapon. Fencing, on the other hand, defends itself and protects prey, which becomes accustomed to domestication.

Early humans, who lived by picking leaves and fruits, naturally discovered the law of "planting and growth" and began to cultivate and plant innovative labor when wild fruits and seeds were scarce. The relationship between weather and planting has been summed up and the cultivation cause has been formed by summing up the growth laws of various plants in planting labor.

Only human beings have the ability to cultivate, but ants grow fungi in their nests. This is also a cultivation. This is an innovation. Ants rely on hidden nests and cultivated fungi to expand the ant colony, but it has halt evolved so far.

\subsection{Inheritance of Language and Culture}

In the productive labor gradually expanded the interpersonal communication, started with the limb sends the signal, the communication more and more, the limb signal has not been able to meet the need. With the evolution of vocal cords and listening, language is formed in communication. The communication of society is the foundation of culture, and language and subsequent writing make the development of culture accumulate and become the actual content of genes. As a result, culture and genes "co-evolved" and passed on from generation to generation. It can be called the inheritance of culture.

From birth to infancy, perhaps from the embryonic stage, people accept the inheritance of human society and culture. The habits and accumulated knowledge of long-term human activity are inherited through genes, on the one hand, and, more importantly, from culture (family and social education, through language, writing, and role models) on the one hand. The fact that cultural inheritance leaves a trail of information in genes can uncover a range of practical examples. A newborn infant can "quack and cry" and "babble"; it takes only a few months to roll and crawl to walk upright; he can learn simple language and speech by the age of one; and music at the age of two or three and read, etc. All this is evidence of cultural heredity. As de Duve [36] points out, "any young ape can't learn to speak, except for some basic gesture language."

There is a genetic basis for cultural inheritance. The scientists studied the genetic changes in several generations of families with language disabilities, suggesting that language disorders are not the common cause of mental retardation, autistic and hearing impairment. Scientists have found that the human gene FOXP2 controls language, and that some families have systemic errors in gene translation and transcription, leading to language disorders in newborns [37-38].

An endless stream of new technologies has been created. New tools have sprung up, such as bones, antlers, ivory and so on, including needles, hooks, darts, spears, bows and arrows. Sew clothes out of animal skin and make buttons with grinding bone rings. Social collaborative actions such as knot notes, barter exchanges, division of labor, and distribution of prey. Make the social culture develop highly.

As an early representative of Homo sapiens, the Cro-Magnons had solemn funeral practices, bodies stained, arms folded, and funerary ornaments, cervical ornaments, weapons or tools. At the same time, the artistic creation has been very rich, including painting and carving, etc. From their totem and murals, it can see that the art of that time has 
reached a very high level.

These fully illustrate the role of culture in human evolution. The evolution of human brain develops social culture, so human culture is the crystallization of the operation of human brain. Regional culture is the collective crystallization of the brainpower of local people. Culture, in turn, promotes a highly developed human brain. Under the influence of human society and culture, mankind entered the era of complete Homo sapiens.

It is quite right to see cultural heredity as a continuation of life. You raise your children, they receive your education, you teach, you leave deep feelings in their young hearts, and they have traces of their genes. That's inheritance. Some people attach importance to genetic inheritance, resentment is not biological, that is, not biological children or parents (that not their own children), but if they understand the human cultural inheritance, will be the comfort of the soul.

The human generation is wiser than the before generation, so-called "Blue comes from the indigo plant but is bluer than the plant itself" which you can directly feel. This is the progress of culture that affects the functioning of the human brain.

\subsection{Mining Excavation and Utilization}

The use of fire expanded early human productivity, and the early sources of energy were mainly wood and, sometimes, solar energy, albeit at low levels.

It was discovered that coal was a good fuel. Clay and glass-pot clay can be used to produce cooked vessels which can be contain food, barbecue and store food, and become an important tool for human beings.

Copper ore and iron ore were discovered in search of raw materials for fired utensils. Mankind walked out of the Neolithic Age and entered the Bronze and Iron Age, which deepened the thinking of scientific invention and creation.

\subsection{Barter}

Social development, originally based on tribal ownership, to meet the needs of the tribe began to barter. The exchange changed the ownership, the powerful man in the tribe stole the goods into private, and the primitive communist ownership of the tribe gradually disintegrated and entered the society with class differentiation.

\section{Conclusion}

The innovative evolution of early human beings fully reflect the laws of natural development and social development. Human development not only adapts to the environment, but also creates a suitable environment for its own development. Adaptation is waiting for change, while innovation is evolution under the stimulation and induction of natural laws. Summing up the above on the early human innovative evolution, reflected mainly in five aspects:

1. Walking upright frees the hands and increases the capacity of the brain to stabilize the head. This is the basis of evolution from apes.

2. Artificial fire is the beginning of the initiative to achieve energy and actively use energy, which is an important aspect of distinguishing other animals.

3. The cooked food obtains the sufficient nutrition, causes the brain function to be perfect. The role of fire and cooked food answers the question of why modern apes cannot evolve into humans.

4. The development of abstract thinking is the deduction of matter. In turn, the substance is transformed. It is impossible for other animals to change the material, which is the fundamental difference between human beings and other animals.

5. It is impossible for any animal to take the initiative to acquire energy and produce food.

\section{References}

[1] Darwin, C., 1872. The Origin of Species. Zhou Jianren, Ye Duzhuang, Fang Zongxi. Triple Bookstore.

[2] Darwin, C., 1872. The Origin of Species. Translated by Peter Shu degan et al. Peking University Press.

[3] Xu Guirong, Wang Yongbiao, Gong Shuyun, Yuan Wei, 2005. Synergetic evolution between organisms and earth environment. China University of Geosciences Press, Wuhan.

[4] Leakey, L. S. B., Tobias, P. V., Napier, J. R., 1964. New species of the genus Homo from Olduvai Gorge. Nature, 202, 7-9.

[5] Leakey, M. G., Spoor, F., Brown, F. H., Gathogo, P. N., Kiarie, C., Leakey, L. N. \& McDougall, I., 2001. New hominin genus from eastern Africa show diverse middle Pliocene lineages. Nature, 410: 433-440.

[6] Wood, B., 2002. Hominid revelations from Chad. Nature, 418:133-135.

[7] Asfaw, B., W. H. Gilbert, Y. Beyene, W. K. Hart, P. R. Renne, G. W. Gabrie, E. S. Vrba, \& T. D. White, 2002. Remains of Homo erectus from Bouri, Middle Awash, Ethiopia. Nature, 416: 317-320.

[8] Wu Xinzh. 1996. The mosaic evolution of humankind in China. In: Glover et. al., eds. Indo-Pacific Prehistory: The Chiang Mai Papers, 2: 225-228. Bulletin of Indo-Pacific Prehistory Association 15.

[9] Walker, A., 1993. The Origin of the Genus Homo. In: Rasmussen, D. T., 1993. The Origin and Evolution of Humans and Humanness. Jones and Bartlett Publisher, London. p. $29-47$

[10] Wolpoff, M. H., Wu, Z., and Thorne, A. G. 1984. Modern Homo sapiens origins: A general theory of hominid evolution involving the fossil evidence from east Asia. In: F. H. Smith and E Spencer (Eds.), The Origins of Modern Humans (New York: Alan R. Liss), pp. 411-483.

[11] Wood, B., 1992. Origin and evolution of the genus Homo. Nature, 355: 783-790.

[12] Carroll, S. B., 2003. Genetics and the making of Homo sapiens. Nature, 422: 849-857. 
[13] Clark, A. G. S. Glanowski, N. Paul, D. Thomas, et al., 2003 Inferring Nonneutral Evolution from Human-Chimp-Mouse Orthologous Gene Trios. Science, 302: 1960-1963.

[14] Diamond, J., 1995. The evolution of human inventiveness. In: Murphy, M. P. \& O'Neill, L. A. J., What is life ? The next fifty years: speculations on the future of biology. Cambridge: Cambridge Univ. Press. 41-55.

[15] Gibbons, A., 1998. Which of Our Genes Make Us Human? Science, Vol. 281, 1432, -1434.

[16] Xu Guirong. The innovative evolution of organisms. In Bao Yueting Editor-in-Chief: long live the motherland-the 60th anniversary of the founding of the excellent collection. China Federation of Literary and Cultural Publishing House 520-522.

[17] Ainsworth, C. et. al., 2005. Life's greatest inventions [J]. New Scientist, 2005 (4), 27-35.

[18] Xu Guirong, Gong Shuyun, Wang Yongbiao, Lin Mingyue, 2012. The Earth's Environment and Biological Innovative Evolution. China University of Geosciences Press, Wuhan.

[19] Xu Guirong, et al. 2013, A collection of papers on the study of the Earth's environment and biological evolution. China University of Geosciences Press, Wuhan.

[20] Xu Guirong 2014. Several problems of biological innovation and evolution. Second, China Radio, Film and Television Publishing House.

[21] Wu Xinzhi, Shang Hongli 2002. Preliminary study on the variation of Homo erectus in China. Quaternary study 22 (1): $20-28$.

[22] Wu Xinzhi. 1990. Evolution of ancient human beings in China. Chinese Journal of Anthropology 9 (4): 312-321.

[23] Wu Xinzhi. 1998a. New progress in the study of human origin. See: Zhang Tao, eds. Science Frontier and Future. Vol. 3. Beijing; Science Press, 161-181.

[24] Wu Mao-lin (1989), Late Homo sapiens in China. In: edited by Wu Rukang, Wu Xinzhi, Zhang Senshui, human beings in Ancient China, Science Press, pp. 42-61.

[25] Wu Rukang, 1995. Thinking about the whole process of human evolution. Journal of Anthropology, vol. 14, No. 4, No. 285-296.

[26] Wu, Xinzh. (2004). "On the origin of modern humans in China”. Quaternary International. 117 (1): 131-140.

[27] Penny, D., 2004. Our relative genetics. Nature, 427: 208-210.

[28] Carmody, Rachel (2009). "The energetic significance of cooking”. Journal of Human Evolution. 57:379-391.

[29] Herman Pontzer, Mary H. Brown, et. al., Metabolic acceleration and the evolution of human brain size and life history, Nature volume 533, pages 390-392 (19 May 2016).

[30] Cho Xi-xi 2014. The age of regular use of fire was enough to shape human civilization, perhaps 350,000 years ago. China Science Journal.

[31] Wrangham, R. 2009. Catching Fire: How Cooking Made Us Human. Basic Books, 2009.

[32] Kimbel, W. H., Donald, C., Rak, Y., 1994. The first skull and other new discoveries of Australopithecus afarensis at Hadar, Ethiopia. Nature, 368: 449-451.

[33] Wu Xinzhi. 1998, The Origin of Modern Chinese from the characteristics of the cranial teeth of the late Homo sapiens in China. Anthropological Journal of Anthropology, 17 (4) 276-282.

[34] Engels, 1876, the role of labor in the transition from ape to man, selected works of Marx and Engels, vol. 3, people's Press, 1975 , pp. 508-520.

[35] Wagner, U., Gals, S., Haider, H., Verleger, R., \& Born, J., 2004. Sleep inspires insight. Nature, 427: 352-354.

[36] de Duve, C., 1992. Vital Dust. the Origin and Evolution of Life on Earth. Trans., Wang Yushan et al., 1999. Vibrant dust-the origin and evolution of life on Earth. Shanghai Science and Technology Education Press.

[37] Lai, C. S. L., Fisher, S. E., Hurst, J. A., Vargha-Khadem, F. \& Monaco, A. P., 2001. A forkhead-domain gene is mutated in a severe speeck and language disorger. Nature, 413:519-523.

[38] Pinker, S., 2001. Talk of genetics and vice versa. Nature, 413:465-466 